Original Paper http://ajol.info/index.php/ijbes http://indexmedicus.afro.who.int

\title{
Etablissement du pollutogramme et de l'hydrogramme de la rivière Kinyankonge, Burundi
}

\author{
Simon BUHUNGU ${ }^{1,2^{*}}$, Arsène M. HOUSSOU ${ }^{2}$, Elie MONTCHOWUI ${ }^{2}$, \\ Gaspard NTAKIMAZI ${ }^{3}$, Jean Luc VASEL ${ }^{4}$ et Théophile NDIKUMANA ${ }^{1}$ \\ ${ }^{1}$ Laboratoire de Chimie et d'Analyses Environnementales, Faculté des Sciences, \\ Université du Burundi (LCAE). B.P. 2700 Bujumbura, Burundi. \\ ${ }^{2}$ Laboratoire de Recherche en Aquaculture et en Biologie et Ecologie Aquatiques, \\ Ecole d'Aquaculture de la Vallée, Université Nationale d'Agriculture, République du Bénin. \\ ${ }^{3}$ Département de Biologie, Faculté des Sciences, Université du Burundi, Burundi. \\ ${ }^{4}$ Facultés des Sciences, Université de Liège, Campus d'Arlon, \\ Avenue de Longwy 185 B - 6700 ARLON, Belgique. \\ *Auteur correspondant ; E-mail: buhusimon@gmail.com
}

\section{REMERCIEMENTS}

Les auteurs remercient l'Académie de Recherche et d'Enseignement Supérieur (l'ARES) de Belgique pour avoir financé cette recherche.

\section{RESUME}

La rivière Kinyankonge, située dans les quartiers Nord-Ouest de la ville de Bujumbura au Burundi, est l'une des six rivières qui traversent cette ville pour se déverser dans le lac Tanganyika. Une étude a été menée pour établir le pollutogramme et l'hydrogramme et rendre compte de l'influence de la station d'épuration de Buterere et des effets anthropiques. Pour ce faire, trois sites de prélèvement ont été choisis. Les prélèvements ont duré quatre mois, d'avril 2012 à juillet 2012 suivant ainsi deux saisons hydrologiques (saison pluvieuse et saison sèche). Les paramètres physico-chimiques ont été déterminés selon les méthodes AFNOR. Les résultats obtenus ont montré qu'à partir du point de déversement des eaux provenant de la station d'épuration, la DCO, la $\mathrm{DBO}_{5}$, le titre alcalimétrique complet, le $\mathrm{pH}$, les ammoniums, les phosphates et les chlorures sont élevés. Quant aux nitrates et nitrites, ils sont élevés à la première station et diminuent pendant la saison sèche. Les matières décantables et les matières en suspension ont augmenté pendant les mois pluvieux (avril et mai). L'hydrogramme de la rivière, mesuré à la première station, a atteint un débit maximum de 152,2 1/s en mai, le minimum étant de 73,9 1/s en juillet. Les flux polluants à cette même station pour les différents paramètres ont varié comme suit: $\mathrm{NH}_{4}{ }^{+}$entre 29,6 et 209,5kg/j; $\mathrm{PO}_{4}{ }^{3-}$ entre 3,4 et 107,8 kg/j; $\mathrm{NO}_{3}{ }^{-}$entre 3,9 et 52,2 $\mathrm{kg} / \mathrm{j} ; \mathrm{NO}_{2}{ }^{-}$ entre 1,3 et $31,1 \mathrm{~kg} / \mathrm{j} ; \mathrm{Cl}^{-}$entre 317,3 et $1307,1 \mathrm{~kg} / \mathrm{j}$; MES entre 480,8 et $12988,8 \mathrm{~kg} / \mathrm{j}$; DCO entre 134,8 et $979,6 \mathrm{~kg} / \mathrm{j}$ et $\mathrm{DBO}_{5}$ entre 74 et $470,2 \mathrm{~kg} / \mathrm{j}$. Ces valeurs ont permis d'établir le pollutogramme de la rivière. L'indice de pollution organique (IPO) a montré que les eaux de la rivière Kinyankonge sont très polluées. (C) 2017 International Formulae Group. All rights reserved.

Mots clés : Débit, flux polluants, Indice de Pollution Organique, rivière Kinyankonge, Burundi. 


\title{
Establishment of the pollutogram and hydrograph of the Kinyankonge River, Burundi
}

\begin{abstract}
The Kinyankonge River, located in the North-Western districts of the city of Bujumbura in Burundi, is one of the six rivers that cross through the city to flow into Lake Tanganyika. A study was carried out to establish the pollutogram and hydrograph and to account for the influence of the Buterere treatment plant and the anthropogenic effects. To achieve this goal, three sampling sites were chosen. The sampling lasted four months, from April 2012 to July 2012, according two hydrological seasons (rainy and dry season). The physicochemical parameters were determined according to the AFNOR methods. The results showed that $\mathrm{COD}, \mathrm{BOD}_{5}$, complete alkalimetric titre, $\mathrm{pH}$, ammoniums, phosphates and chlorides were high from the point of discharge of the wastewater from the treatment plant of Buterere. As for nitrates and nitrites, they are higher at the first station and decrease during the dry season. Decanting materials and suspended solids increased during the rainy months (April and May). The hydrograph of the river, measured at the first station, reached a maximum flow of $152.21 / \mathrm{s}$ in May, the minimum being $73.91 / \mathrm{s}$ in July. The pollutant fluxes at the same station for the various parameters varied as followed: $\mathrm{NH}_{4}{ }^{+}$between 29.6 and $209.5 \mathrm{~kg} / \mathrm{d}$; $\mathrm{PO}_{4}{ }^{3-}$ between 3.4 and $107.8 \mathrm{~kg} / \mathrm{d} ; \mathrm{NO}_{3}{ }^{-}$between 3.9 and $52.2 \mathrm{~kg} / \mathrm{d} ; \mathrm{NO}_{2}^{-}$between 1.3 and $31.1 \mathrm{~kg} / \mathrm{d} ; \mathrm{Cl}^{-}$between 317.3 and $1307.1 \mathrm{~kg} / \mathrm{d}$; MES between 480.8 and $12988.8 \mathrm{~kg} / \mathrm{d}$; COD between 134.8 and $979.6 \mathrm{~kg} / \mathrm{d}$ and BOD $_{5}$ between 74 and $470.2 \mathrm{~kg} / \mathrm{d}$. These values were used to establish the river pollutogram. The organic pollution index (OPI) has shown that the waters of the Kinyankonge River are highly polluted.
\end{abstract}

(C) 2017 International Formulae Group. All rights reserved.

Keywords: Flow rate, pollutant, Organic Pollution Index, Kinyankonge River, Burundi.

\section{INTRODUCTION}

Les eaux douces sont parmi les écosystèmes les plus sérieusement menacés par l'impact des effets anthropiques au cours de ce dernier siècle (Sala et al., 2000; Cox et al., 2002 ; Dudgeon et al., 2006). Pourtant, dans la majorité des pays en développement, la plupart des cours d'eau urbains sont utilisés pour l'évacuation des déchets des villes et des industries. Il s'en suit une dégradation de la qualité de l'eau, une modification des peuplements et, le plus souvent, une diminution de la biodiversité (Togouet, 2008). La rivière Kinyankonge, traversant la ville de Bujumbura, dans ses quartiers Nord-Ouest, subit tout au long de son parcours des pressions anthropiques. Située dans la zone d'inondation, la rivière reçoit les eaux de ruissellement pluviales provenant des sols dégradés à cause des pratiques agricoles non adaptées sur des fortes pentes et à une déforestation en amont, ce qui a été à l'origine de la catastrophe qui a causé des dégâts aux dates des 9-10 février 2014. En plus, les rejets domestiques, les rejets d'une savonnerie constituent des sources de pollution pour cette rivière et une menace pour la qualité de l'eau et la biodiversité de cette rivière et du lac Tanganyika dont elle est l'affluente. Ainsi, la qualité physico-chimique et biologique du milieu est affectée (Houssou et al., 2015).

Outre les rejets directs, la rivière Kinyankonge reçoit les eaux provenant d'une station d'épuration (STEP) dans la zone urbaine de Buterere. Les STEP sont conçues pour épurer les eaux usées et limiter ainsi l'apport en excès de matière organique et de polluants dans le milieu naturel (Vindimian, 2006). Mais plusieurs études ont montré que les rejets des stations d'épuration ont différents niveaux de charge polluante selon l'efficacité de la STEP. Dans certains pays, les eaux usées traitées sont réutilisées pour l'irrigation des champs de façon que les projets ont investi dans ce domaine et plus récemment en Australie et en Afrique du Sud (Mohammad et Mazareh, 2003 ; AQUEREC Project, 2006 ; Salgot et Huertas, 2006).

Niyungeko (2011) et Nsavyimana (2014) ont rapporté que la rivière 
Kinyankonge est un canal de transfert des polluants provenant de la STEP vers le lac Tanganyika. La présente étude vise à évaluer l'état de pollution et la structure hydrographique de la rivière Kinyankonge pendant les saisons pluvieuses et sèches.

\section{MATERIEL ET METHODES}

Trois stations d'échantillonnage ont été choisies (Figure 1) dans le cadre de la présente étude. La première station ( $\mathrm{S} 1$, latitude S $3^{\circ} 20^{\prime} 42,623 "$, longitude E 29²1'11,275", altitude de $771.31 \mathrm{~m})$ est positionnée en amont tout juste après l'embouchure de la rivière Nyabagere sur la Kinyankonge. La deuxième station (S2, latitude S 3०21'16,657", longitude E $29^{\circ} 20^{\prime} 32,535^{\prime \prime}$, d'altitude $764.56 \mathrm{~m}$ ) a été choisie juste après le point de rejet de la STEP dans la Kinyankonge. Cette station d'épuration est sous-utilisée puisqu'elle ne traite que les eaux usées de 5 zones sur les 13 zones des trois communes de la Mairie de Bujumbura. La troisième station $(\mathrm{S} 3$, latitude S 3²1'37,346", longitude E 29²0'22,794", altitude $760.60 \mathrm{~m}$ ) quant à elle est choisie en aval un peu avant l'embouchure de la Kinyankonge sur le Lac Tanganyika. Elle reçoit en plus les rejets en provenance de l'usine de fabrication des savons. Ces stations ont été choisies en raison de la diversité des rejets. Au Burundi, la saison sèche dure trois mois (juin-juillet-août) et la saison pluvieuse le reste de l'année c'est-à-dire de septembre à mai (Ntakiyiruta et al., 2017). L'analyse des paramètres tels que le débit, les nutriments, les chlorures, les matières en suspension, la demande chimique et biologique en oxygène permettront d'évaluer l'état de la pollution. La détermination du débit permettra le calcul du flux polluant.

\section{Mesure in-situ et échantillonnage d'eau}

Les mesures des paramètres physicochimiques et les prélèvements d'eau sur terrain ont duré quatre mois, d'avril à juillet. Deux paramètres ont été mesurés sur le terrain : le $\mathrm{pH}$ et le débit. Le $\mathrm{pH}$ est mesuré à l'aide d'un $\mathrm{pH}$-mètre portable de marque Consort C 6010. Le débit, quant à lui, a été mesuré à la première station pour des raisons d'accessibilité, en utilisant l'injecteur à bulles selon la méthode développée par Dufayt et al. (1994). Le principe consiste à injecter une bulle au fond du cours d'eau. Celle-ci va être animée d'un mouvement translationnel associé à la vitesse du courant et d'un mouvement ascensionnel. La distance parcourue par la bulle entre le point d'injection et son point d'émergence est mesurée chaque $20 \mathrm{~cm}$ sur la largeur du cours d'eau. Les différentes distances mesurées sont utilisées pour déterminer le débit par intégration du champ des vitesses sur la verticale correspondante.

Pour les autres analyses, sur chacune des trois stations, un échantillon d'eau (de surface) a été prélevé entre 10 et $15 \mathrm{~cm}$ de profondeur, puis conditionné dans des bidons en plastiques de cinq litres. Les bidons préalablement nettoyés étaient rincés avec l'eau de la station avant le remplissage. Cette eau brute de la rivière était immédiatement conservée dans des glaçons (durant le transport) puis au réfrigérateur $\left(4{ }^{\circ} \mathrm{C}\right)$ pour l'analyse des autres paramètres physicochimiques au Laboratoire de Chimie et d'Analyses Environnementales (LCAE) de la Faculté des Sciences à l'Université du Burundi. Les prélèvements d'échantillons d'eau se faisaient de 10 à 12 heures.

\section{Méthodes d'analyse au laboratoire Matières décantables}

Les matières décantables ont été déterminées par le cône Imhof selon la méthode de Rodier (2005). Les résultats ont été exprimés en millilitres de matières décantées pour un litre d'eau.

\section{Matières en suspension}

La détermination des matières en suspension dans l'eau a été effectuée par filtration sur membrane en fibres de verre selon la Norme AFNOR, NFT90- 105 (Rodier, 2005).

\section{Alcalinité}

L'alcalinité a été déterminée par volumétrie selon les méthodes APHA (1995) et AFNOR NTF 90-003 (Rodier, 2005). Cent millilitres d'échantillon sont dosés par de 
l'acide chlorhydrique $0,02 \mathrm{~N}$ en présence de méthylorange (indicateur d'équivalence). Le point d'équivalence est atteint lorsque la solution passe de l'orange au jaune orangé.

\section{Demande chimique en oxygène (DCO)}

La demande chimique en oxygène a été déterminée selon la méthode normalisée AFNOR (T90-101). Elle s'est déroulée en deux étapes. Une étape de la minéralisation dans un bloc chauffant avec des réactifs prédosés $\left(\mathrm{H}_{2} \mathrm{SO}_{4}, \mathrm{Ag}_{2} \mathrm{SO}_{4}, \mathrm{~K}_{2} \mathrm{SO}_{4}\right.$ et $\left.\mathrm{K}_{2} \mathrm{Cr}_{2} \mathrm{O}_{7}\right)$. Deux millilitres d'échantillon ont été additionnés de réactifs et portés à $148{ }^{\circ} \mathrm{C}$ pendant 2 heures dans un minéralisateur. La seconde étape a été celle de la mesure dans un photomètre PalinTest modèle DR 7500 à une longueur d'onde de $490 \mathrm{~nm}$.

\section{Demande biochimique en oxygène $\left(\mathrm{DBO}_{5}\right)$}

La demande biochimique en oxygène $a$ été mesurée à l'aide d'un DBO-mètre. Un volume d'eau correspondant à la gamme d'estimation de la $\mathrm{DBO}_{5}$ par rapport à la DCO du même échantillon a été versé dans le flacon en verre brun contenant un barreau magnétique. Deux pastilles de soude caustique $(\mathrm{NaOH})$ ont été ensuite introduites dans le godet en caoutchouc. La soude caustique a pour rôle d'absorber le $\mathrm{CO}_{2}$ produit lors de l'oxydation des matières organiques. $\mathrm{La}$ quantité d'oxygène est enregistrée toutes les 24 heures par l'OxiTop ${ }^{\mathrm{R}}$ sur une période de 5 jours. La $\mathrm{DBO}_{5}$ exprimée en $\mathrm{mg} \mathrm{O}_{2} / 1$ est obtenue par multiplication de la valeur enregistrée au jour 5 par un facteur déterminé selon la gamme de DCO.

Dosage des orthophosphates, des ammoniums, des nitrates et des nitrites

Les orthophosphates, les ammoniums, les nitrates et les nitrites ont été dosés par photométrie en utilisant le photomètre PalinTest modèle DR 7500, avec des réactifs pré-dosés à des longueurs d'onde différentes (640 $\mathrm{nm}$ pour les phosphates, $420 \mathrm{~nm}$ pour les ammoniums, $543 \mathrm{~nm}$ pour les nitrites et 415 $\mathrm{nm}$ pour les nitrates).

Les ions nitrates ont été mesurés par colorimétrie en utilisant un photomètre PalinTest DR 7500 sur un échantillon d'eau. Le choix du programme Phot 88 a permis de sélectionner la longueur d'onde de $415 \mathrm{~nm}$ pour le dosage des nitrates. Les résultats ont été affichés en $\mathrm{mg} / \mathrm{l}$ de $\mathrm{NO}_{3}{ }^{-}$.

Quant aux nitrites, ces derniers réagissent avec l'acide sulphanilique en milieu acide pour former le N-(1-Naphthyl)-éthylène diamine dont l'intensité de couleur pourpre est proportionnelle à la concentration en nitrites. Le choix du programme Phot 24 a permis de sélectionner la longueur d'onde de $543 \mathrm{~nm}$ pour le dosage des nitrites dans l'échantillon donné. Les résultats ont été affichés en $\mathrm{mg} / \mathrm{l}$ de $\mathrm{NO}_{2}^{-}$(Rodier et al., 2009).

\section{Chlorures}

Les chlorures ont été dosés par argentimétrie (Rodier et al., 2009). Il s'agit d'un dosage en milieu neutre par une solution titrée de nitrate d'argent en présence de chromate de potassium. La fin de la réaction est indiquée par l'apparition de la teinte rouge brique caractéristique du chromate d'argent.

\section{Traitement des données}

La statistique descriptive a été appliquée aux données. La moyenne \pm écarttype de chaque paramètre a été comparée entre les stations et dans le temps avec le test Anova à un critère de classification (ANOVA 1) en utilisant le logiciel STATISTICA 7.1 au seuil de $5 \%$.

Le flux polluant dans la première station a été déterminé par la relation $F(\mathrm{~kg} / \mathrm{j})$ $=\mathrm{Q}_{\mathrm{i}}{ }^{*} \mathrm{C}_{\mathrm{i}}{ }^{*} \Delta \mathrm{T}_{\mathrm{i}}$, avec $\mathrm{Q}_{\mathrm{i}}$ le débit exprimé en litres par seconde, $\mathrm{C}_{\mathrm{i}}$ la concentration du polluant en milligrammes par litre et $\Delta \mathrm{T}_{\mathrm{i}}$ le temps en jours (24 heures).

L'Indice de Pollution Organique (IPO) de Leclercq (2001) a été également calculé pour évaluer la charge organique dans la rivière. Cet indice s'obtient au moyen des valeurs ammoniums, la $\mathrm{DBO}_{5}$, nitrites et phosphates. Le principe du calcul est de répartir les valeurs des quatre éléments polluants en cinq classes et de déterminer à partir des valeurs obtenues dans l'étude, le numéro de classe correspondant pour chaque paramètre en se servant des données moyennes du Tableau 1. L'indice final est la moyenne des classes de pollution pour l'ensemble des paramètres. 


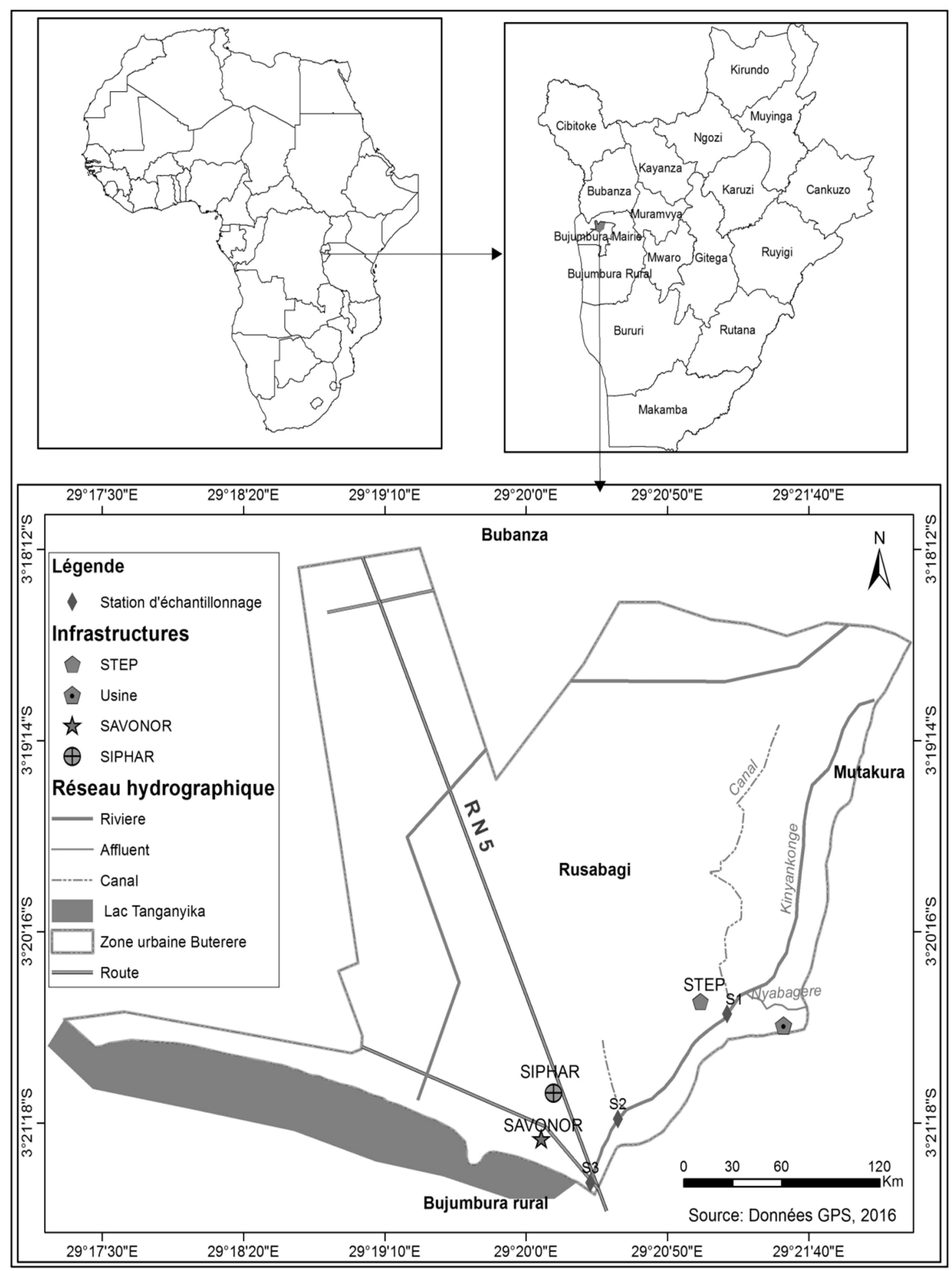

Figure 1: Carte géographique des stations d'étude sur la rivière Kinyankonge. 
Tableau 1 : Limites des classes de l'Indice de Pollution Organique (Leclercq, 2001).

\begin{tabular}{lccccc}
\hline Classes & $\mathbf{N H}_{4}+(\mathbf{m g} / \mathbf{l})$ & $\begin{array}{c}\mathbf{D B O}_{\mathbf{5}} \\
\left.\mathbf{m g O}_{2} / \mathbf{l}\right)\end{array}$ & $\mathbf{N O}_{2}-(\boldsymbol{\mu g} / \mathbf{l})$ & $\mathbf{P O}_{4}{ }^{3-}(\boldsymbol{\mu g} / \mathbf{l})$ & $\begin{array}{c}\text { Classe de } \\
\text { qualité IPO }\end{array}$ \\
\hline 5 & $<0,1$ & 2 & $<5$ & $<15$ & $4,6-5,0$ \\
4 & $0,1-0,9$ & $2,1-5$ & $6-10$ & $16-75$ & $4,0-4,5$ \\
3 & $1-2,4$ & $5,1-10$ & $11-50$ & $76-250$ & $3,0-3,9$ \\
2 & $2,5-6$ & $10,1-15$ & $51-150$ & $251-900$ & $2,0-2,9$ \\
1 & $>6$ & $>15$ & $>150$ & $>900$ & $1,0-1,9$ \\
\hline (5) Pollution organique nulle, (4) pollution organique faible, (3) pollution organique modérée, (2) pollution organique \\
forte, (1) pollution organique très forte.
\end{tabular}

\section{RESULTATS}

\section{Variabilité temporelle des paramètres} physico-chimiques

Une différence significative a été notée dans la charge en ammonium de la rivière durant la période d'étude $(\mathrm{p}<0,05)$. Une évolution croissante a été observée d'avril à juillet pour ce paramètre. Pour les nitrites et les nitrates, la variation a également été significative $(\mathrm{p}<0,05)$. Le mois de mai a été caractérisé par une plus grande charge pour le compte de ces deux paramètres et le mois de juillet a enregistré une faible pollution. De même pour les phosphates, une différence significative a été notée avec une plus grande charge en avril et une concentration plus faible en juillet. Les chlorures ont varié significativement $(\mathrm{p}<0,05)$ avec une plus grande concentration en mai et une plus faible en avril. Pour les paramètres, MES, matières décantables, $\mathrm{DCO}$ et $\mathrm{DBO}_{5}$, aucune différence significative n'a été notée ( $p>$ $0,05)$.

$\mathrm{Au}$ regard du titre alcalimétrique complet, une différence significative a été notée ( $\mathrm{p}<0,05)$. L'évolution de ce paramètre a été croissante d'avril à juillet. Il en est de même pour le $\mathrm{pH}$ où une différence significative a été enregistrée entre les mois avec la plus petite moyenne en avril et la moyenne élevée en mai (Tableau 2).

\section{Caractérisation spatiale des paramètres physico-chimiques}

Aucune différence significative n'a été notée pour les ammoniums, les phosphates, les nitrates, les nitrites, les chlorures, les matières décantables, le TAC et le $\mathrm{pH}$ entre les stations échantillonnées ( $p>0,05)$. Pour les MES, une différence significative a été observée $(\mathrm{p}<0,05)$. La plus grande moyenne a été observée à la troisième station et la plus petite moyenne a été notée à la deuxième station. $\mathrm{La} \mathrm{DCO}$ et la $\mathrm{DBO}_{5}$ ont significativement varié entre les stations. Une évolution croissante a été observée pour ces deux paramètres de la première à la troisième station (Tableau 3).

\section{Variabilité spatio-temporelle de la charge organique}

Le Tableau 4 présente les variations spatio-temporelles de l'indice de pollution organique. Les valeurs trouvées pour IPO sont comprises entre 1,0 -1,25. 
Hydrogramme de la rivière Kinyankonge: Station 1

Les valeurs de débit enregistrées à différentes dates sont présentées sur la Figure 2. Les pics observés sur le graphique correspondent aux évènements pluvieux observés aux mois de mai avec 152,2 1/s et de juin avec 135,4 1/s. Les minima, quant à eux, correspondent au débit de la saison sèche et ont été observés en juin, avec 78,3 1/s et juillet avec, 73,9 1/s.
Variabilité temporelle du flux polluant à la première station

Le flux polluant moyen pendant la période d'étude est présenté à la Figure 3 . Pour l'ensemble des paramètres (ammoniums, nitrates, nitrites, phosphates, chlorures, matières en suspension, $\mathrm{DCO}$ et $\mathrm{DBO}_{5}$ ) à l'exception de la $\mathrm{DBO}_{5}$ et la DCO, le mois de mai a été la période des grands flux. Dans le cas de ces deux paramètres (la $\mathrm{DBO}_{5}$ et la DCO), c'est plutôt pendant le mois d'avril que les plus grands flux ont été observés. Seuls les nitrates et les chlorures ont présenté des flux significativement différents dans la période d'étude.

Tableau 2 : Variabilité temporelle des paramètres physico-chimiques.

\begin{tabular}{|c|c|c|c|c|}
\hline Paramètres & Avril & Mai & Juin & Juillet \\
\hline $\mathrm{NH}_{4}{ }^{+}(\mathrm{mg} / \mathrm{L})$ & $3,24 \pm 0,67^{\mathrm{a}}$ & $7,28 \pm 1,97^{b}$ & $11,14 \pm 4,00^{\mathrm{c}}$ & $11,51 \pm 2,19^{\mathrm{c}}$ \\
\hline $\mathrm{PO}_{4}{ }^{3-}(\mathrm{mg} / \mathrm{L})$ & $2,90 \pm 1,02^{\mathrm{a}}$ & $1,29 \pm 0,39^{b}$ & $1,23 \pm 0,48^{\mathrm{b}}$ & $1,05 \pm 0,38^{\mathrm{b}}$ \\
\hline $\mathrm{NO}_{3}^{-}(\mathrm{mg} / \mathrm{L})$ & $1,32 \pm 0,41^{\mathrm{a}}$ & $1,97 \pm 0,69^{b}$ & $1,01 \pm 0,50^{\mathrm{ac}}$ & $0,62 \pm 0,36^{\mathrm{c}}$ \\
\hline $\mathrm{NO}_{2}^{-}(\mathrm{mg} / \mathrm{L})$ & $0,45 \pm 0,16^{\mathrm{a}}$ & $0,47 \pm 0,20^{\mathrm{a}}$ & $0,37 \pm 0,26^{\mathrm{ab}}$ & $0,21 \pm 0,04^{\mathrm{b}}$ \\
\hline $\mathrm{Cl}^{-}(\mathrm{mg} / \mathrm{L})$ & $60,62 \pm 9,86^{\mathrm{a}}$ & $88,17 \pm 18,04^{\mathrm{b}}$ & $66,14 \pm 12,97^{\mathrm{a}}$ & $62,91 \pm 13,68^{\mathrm{a}}$ \\
\hline $\operatorname{MES}(\mathrm{mg} / \mathrm{L})$ & $125,50 \pm 49,09^{\mathrm{a}}$ & $134,06 \pm 58,24^{\mathrm{a}}$ & $129,86 \pm 34,53^{\mathrm{a}}$ & $97,00 \pm 20,06^{\mathrm{a}}$ \\
\hline Mat.déc(ml/L) & $0,23 \pm 0,17^{\mathrm{a}}$ & $0,27 \pm 0,19^{\mathrm{a}}$ & $0,22 \pm 0,10^{\mathrm{a}}$ & $0,19 \pm 0,13^{\mathrm{a}}$ \\
\hline $\mathrm{DCO}(\mathrm{mgO} 2 / \mathrm{L})$ & $59,11 \pm 22,07^{\mathrm{a}}$ & $53,78 \pm 23,27^{\mathrm{a}}$ & $74,58 \pm 36,75^{\mathrm{a}}$ & $85,47 \pm 59,02^{\mathrm{a}}$ \\
\hline $\mathrm{DBO}_{5}\left(\mathrm{mgO}_{2} / \mathrm{L}\right)$ & $32,33 \pm 11,79^{\mathrm{a}}$ & $26,67 \pm 11,99^{\mathrm{a}}$ & $38,33 \pm 19,35^{\mathrm{a}}$ & $38,67 \pm 20,29^{a}$ \\
\hline TAC(méq/L) & $3,82 \pm 2,03^{\mathrm{a}}$ & $5,68 \pm 2,07^{\mathrm{ab}}$ & $7,54 \pm 1,51^{\mathrm{bc}}$ & $9,35 \pm 2,72^{\mathrm{c}}$ \\
\hline pH & $7,40 \pm 0,11^{\mathrm{a}}$ & $7,75 \pm 0,15^{\mathrm{b}}$ & $7,65 \pm 0,17^{b}$ & $7,70 \pm 0,10^{\mathrm{b}}$ \\
\hline
\end{tabular}


S. BUHUNGU et al. / Int. J. Biol. Chem. Sci. 11(3): 1386-1399, 2017

Tableau 3 : Variabilité spatiale des paramètres physico-chimiques.

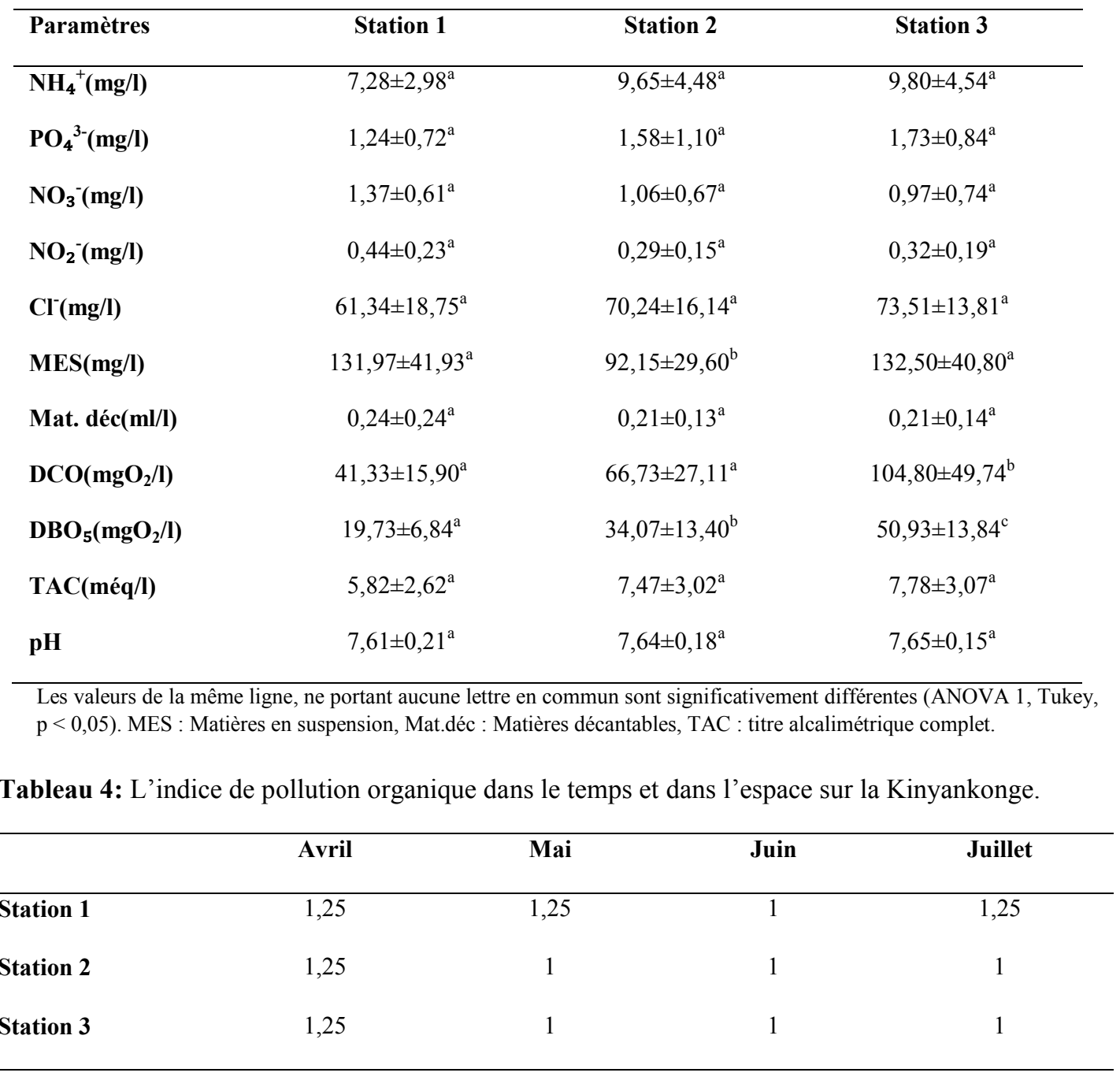

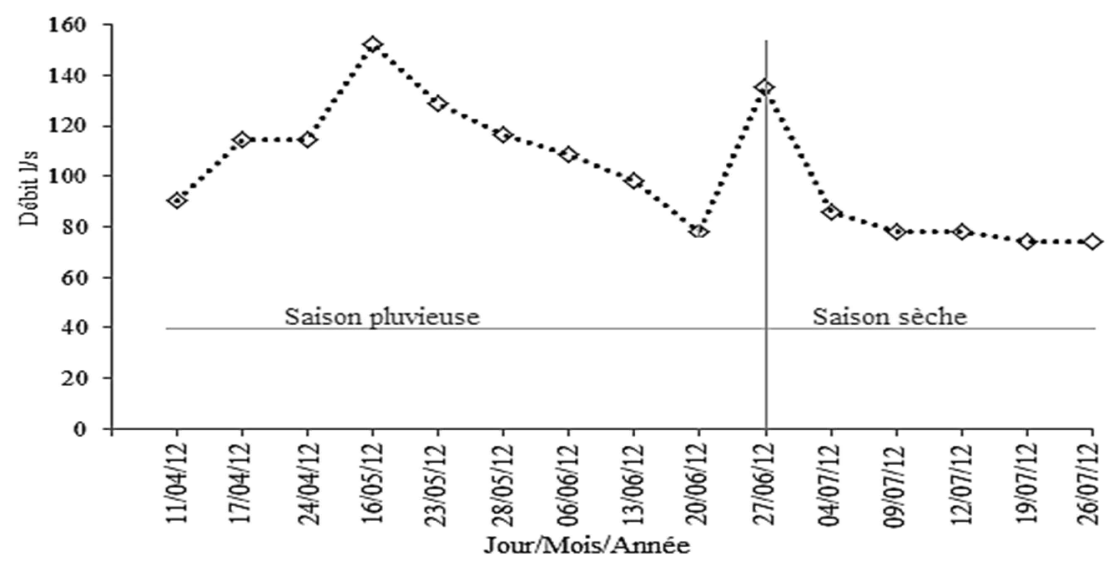

Figure 2: Hydrogramme de la rivière Kinyankonge d'avril 2012 à mai 2012. 
S. BUHUNGU et al. / Int. J. Biol. Chem. Sci. 11(3): 1386-1399, 2017
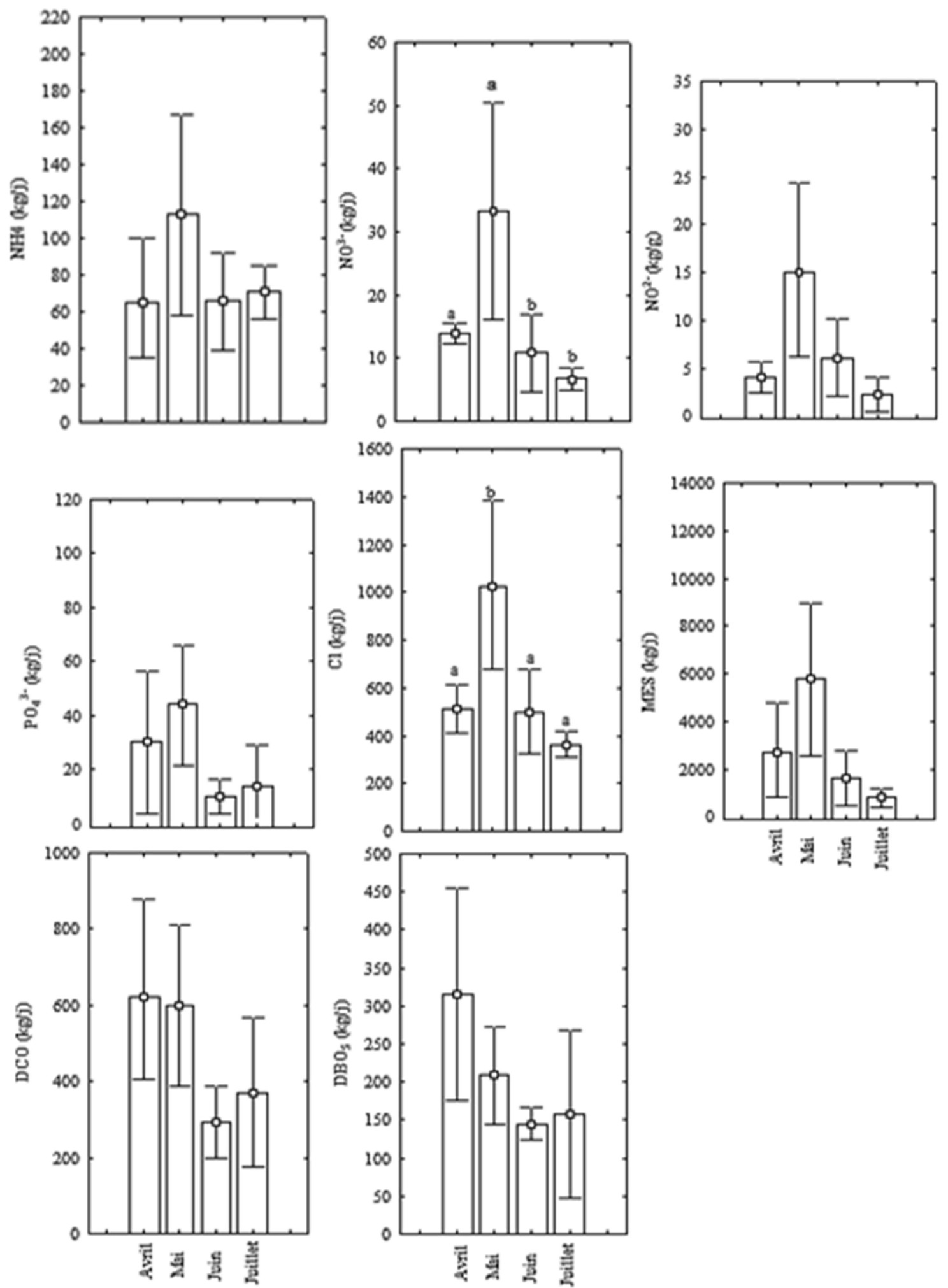

Figure 3: Variation des flux polluants dans la rivière Kinyankonge. 


\section{DISCUSSION}

Les résultats issus de la mesure du débit au cours de la présente étude ont permis d'établir l'hydrogramme de la rivière Kinyankonge pendant la saison pluvieuse et sèche (Figure 2). Le débit mesuré pendant la période sèche a été de 73,91 /s, soit 6384,96 $\mathrm{m}^{3}$ par jour. Ce débit est inférieur à celui obtenu (7330 $\mathrm{m}^{3}$ par jour) par Niyungeko (2011) à la même station et à la même période. Normalement, au Burundi, la saison sèche dure trois mois (juin, juillet et août) et la saison pluvieuse le reste des mois de l'année (Ntakiyiruta et al., 2017). Dans la présente étude, la saison pluvieuse s'est prolongée jusqu'au mois de juin. Ceci se remarque sur l'hydrogramme par le pic enregistré le 27 juin 2012. Des évènements pluvieux accidentels avaient, en effet, été notés, et cela est courant dans le cadre de la variabilité climatique naturelle. Le débit élevé, enregistré au mois de mai, est consécutif aux fortes pluies de la saison pluvieuse généralement en cours pendant ce mois au Burundi. Les mêmes observations ont été faites par Bakundukize et al. (2011) pour lesquelles, près de la moitié des précipitations interviennent entre février et mai.

Certains paramètres physico-chimiques ayant servi à l'établissement du pollutogramme ont varié aussi bien pendant la saison pluvieuse que la saison sèche (Tableaux 2). La variation de ces paramètres physico-chimiques peut être attribuée à la charge polluante provenant des déchets domestiques et des activités agricoles le long du bassin de la rivière. En effet, lors des épisodes pluvieux, divers déchets entreposés dans le bassin versant de la Kinyankonge sont entraînés par les eaux de ruissellement avec d'importantes quantités de matières organiques dans la rivière. Outre les rejets domestiques urbains, les polluants d'origine agricole ont été considérés comme sources de pollution (Majaliwa et al., 2004; Azanga et al., 2016).

Des écarts pour certains paramètres ont été relevés entre les saisons. Il s'agit des ammoniums, des phosphates, des nitrates, des nitrites et des chlorures. Des observations similaires qui expliquent de fortes variations entre les saisons ont été rapportées sur les rivières Kahuwa et Mpungwe en République Démocratique du Congo (Zirirane et al., 2014; Bagalwa et al., 2012).

Les flux polluants en ammoniums relevés dans cette étude sont plus élevés par rapport à ceux des nitrates et des nitrites. Des études similaires, menées sur la rivière Ntahangwa, affluente du lac Tanganyika, ont abouti au même constat (Brion et al., 2006). Plusieurs auteurs (Ansa-Assar et al., 2000; Kotak et al. 2000; Downing et al., 2001; Sondergaard et al., 2003; Li et al., 2009) s'accordent sur le fait que de nombreuses activités humaines impliquent une concentration de nutriments, notamment du phosphore et de l'azote, sur un nombre restreint de bassins versants. Les nitrates et les nitrites ont augmenté au mois de mai, correspondant aux fortes pluies, ce qui a fait que la charge polluante calculée à la première station à cette période pour ces deux paramètres était élevée (Figure 3). En plus des rejets domestiques issus d'activités anthropiques, la présence des nitrates et des nitrites dans les cours d'eau est due, soit au lessivage des terres agricoles, soit aux réactions oxydatives de l'azote ammoniacal et des nitrites (Saad et al., 2004; Khalaf et al., 2007, Ntakiyiruta et al., 2017). Les fortes valeurs de nitrates ont été enregistrées au mois de mai pendant les périodes pluvieuses, tandis que les faibles valeurs ont été enregistrées aux mois de juin et juillet correspondant à la saison sèche. Les mêmes observations ont été faites par Ntakiyiruta et al. (2017) sur la rivière Ntahangwa. Les intenses activités agricoles observées le long de la rivière Kinyankonge utilisent, en effet, des engrais azotés et sont source des nitrates.

En ce qui concerne les phosphates, des teneurs élevées sont enregistrées aux mois d'avril et de mai. La présence des composés phosphorés dans une rivière est traduite bien souvent par le rejet d'eaux usées domestiques. En effet, beaucoup de produits domestiques d'entretien tels que les savons et les détergents 
contiennent des éléments phosphorés (Jen 2002). Les valeurs enregistrées dans cette étude se rapprochent de celles de Nassali et al. (2005) qui ont enregistré une teneur comprise entre 0,90 et $3,56 \mathrm{mg} / \mathrm{l}$ sur le lac Merja Fouarate au Maroc.

Les matières en suspension représentent l'ensemble des particules minérales et organiques contenues dans les eaux. Elles sont fonction de la nature des terrains traversés, de la saison, de la pluviométrie, du régime d'écoulement des eaux, de la nature des rejets. Les teneurs élevées en matières en suspension sont considérées comme une forme de pollution. Selon Pitt et al. (2005) et Wong (2006), plusieurs polluants peuvent être associés au ruissellement comme les métaux toxiques et les nutriments se liant avec les particules de sédiments fins avec une incidence négative sur le milieu récepteur. Les résultats enregistrés sont dans les limites acceptables d'intervalle de variation de quelques paramètres de pollution des eaux de ruissellement de 150 à $350 \mathrm{mg} / \mathrm{l}$ pour les matières en suspension. Par contre, des MES de plus de $35 \mathrm{mg} / \mathrm{l}$ dépassent de loin la norme de rejet (Normes Internationales fixées entre 20-30 mg/l). Les matières en suspension sont élevées à la première et à la troisième station. Ce qui montre que la station d'épuration de Buterere déverse pratiquement des effluents non traités dans la Kinyankonge. Le même constat a été fait par Niyungeko (2011) et Nsavyimana (2014) qui ont estimé que cette station constitue une source de pollution pour la rivière puisqu'une partie de la charge polluante non traitée y est déversée; elle ne fonctionne pas correctement. D'où la nécessité de raccorder toutes les zones de la Mairie de Bujumbura afin d'avoir un débit suffisant à traiter.

La demande chimique en oxygène de la rivière Kinyankonge a demeuré globalement en dessous de la norme admise de $80 \mathrm{mg} \mathrm{O} \mathrm{O}_{2} / 1$ selon Bliefert et Perraud (2001). $\mathrm{La} \mathrm{DBO}_{5}$ est aussi une mesure de la capacité d'autoépuration d'un cours d'eau; si sa valeur est au plus égale à $3 \mathrm{mgO}_{2} / \mathrm{l}$, l'eau est qualifiée d'excellente qualité. Les moyennes de $\mathrm{DBO}_{5}$ relevées ont montré que les valeurs sont toujours supérieures aux normes de rejet fixées à $25 \mathrm{mgO}_{2} / 1$, exceptée, la station $\mathrm{S} 1$ où une moyenne de $19,73 \mathrm{mgO}_{2} / 1$ a été enregistrée. L'eau est ainsi située dans la catégorie «hors classe» (Bliefert et Perraud, 2001). Ces deux paramètres ont évolué de l'amont vers l'aval (Tableau 3). Ce qui signifie que sur son parcours, la Kinyankonge reçoit des rejets d'origines diverses dans les quartiers qu'elle traverse. Ce constat a aussi été fait par Ntakiyiruta et al. (2017) sur la rivière Ntahangwa.

Les chlorures sont des anions inorganiques importants contenus en concentrations variables dans les eaux naturelles, généralement sous forme de sels de sodium $(\mathrm{NaCl})$ et de potassium $(\mathrm{KCl})$. Ils sont souvent utilisés comme un indice de pollution. Ils ont une influence sur la faune et la flore aquatique et sur la croissance des végétaux. En plus, ils augmentent lors d'un évènement pluvieux, suite à un lessivage en provenance des produits chlorés déversés dans la rivière pendant la période pluvieuse.

Les eaux usées issues de la savonnerie SAVONOR proviennent du lavage de soapstock fortement chargés en matières organiques dues aux souillures contenues dans la matière première. Selon Drieu et al. (2000), dans son rapport technique sur la lutte contre la pollution et autres mesures visant à protéger la biodiversité du Lac Tanganyika, les produits contenant du $\mathrm{NaOH}$ sont déversés dans la rivière Kinyankonge après nettoyage des cuves contribuant à l'augmentation du pH.

Les valeurs moyennes de l'IPO calculées à chaque station et à la période considérée sont très basses (Tableau 4). Ces valeurs s'expliquent par les teneurs très élevées en ammoniums, en phosphates, en nitrites et en $\mathrm{DBO}_{5}$. L'évolution des valeurs de l'IPO le long des stations montre que la dégradation des eaux de la Kinyankonge est due aux rejets des eaux usées domestiques et de la STEP provenant des zones urbaines de Buterere et Cibitoke riveraines de la rivière. Le même constat a été fait par Ben Bouih et 
al. (2005) sur d'autres rivières. Ainsi, l'indice de pollution organique appliqué à ces paramètres a montré que les eaux de la rivière Kinyankonge sont fortement polluées. Elles sont ainsi qualifiées de mauvaise qualité (Leclercq, 2001).

\section{Conclusion}

Certains paramètres physico-chimiques des eaux de la rivière Kinyankonge présentent des valeurs qui dépassent les normes de rejet dans le milieu récepteur final qui est le lac Tanganyika. Ainsi, elles sont classées dans la catégorie des eaux polluées (Leclercq, 2001). Ce qui présente un danger public pour les habitants localisés dans la zone urbaine de Buterere et pour les espèces de la faune et de la flore qui vivent dans cet écosystème aquatique. Des études approfondies couvrant au moins une année et plusieurs saisons hydrologiques sont nécessaires pour mettre en exergue les effets néfastes de cette pollution et développer des outils de surveillance et de biomonitoring de la rivière Kinyankonge, en particulier et du Lac Tanganyika en général.

\section{CONFLIT D'INTERETS}

Les auteurs de cet article déclarent qu'il n'y a aucun conflit d'intérêts pour cette publication.

\section{CONTRIBUTION DES AUTEURS}

$\mathrm{SB}, \mathrm{TN}$ ont réalisé la conception de l'étude, la collecte des données et l'analyse au laboratoire; SB, $\mathrm{AMH}, \mathrm{TN}$ et JLV ont participé à l'analyse des données; la rédaction de l'article a été par SB, AMH, EM, GN, TN et JLV.

\section{RÉFÉRENCES}

Ansa-Asare OD, Marr IL, Cresser MS. 2000. Evaluation of modelled and measured patterns of dissolved oxygen in a freshwater lake as an indicator of the presence of biodegradable organic pollution. Water Research, 34(4): 10791088.

Doi:10.1016/S0043-1354
APHA (American Public Health Association). 1995. Standard Methods for the Examination of Water and Waste Water, $\left(19^{\text {th }}\right.$ edn). APHA: Washington D.C., USA; 874.

AQUEREC Project. 2006. Work package 2 final report, Delivrable D15, EVK1-CT20026-00130. Integrated concepts for reuse of upgraded wastewater. URL WWW.aquerec.org.

Azanga E, Majaliwa M, Kansiime F, Mushagalusa N, Korume $\mathrm{K}$, Tenywa MM. 2016. Land-use and land-cover, sediment and nutrient hotspot areas changes in Lake Tanganyika basin. African Journal of Rural Development, 1(1): 75-90.

Bagalwa M, Zirirane N, Pauls S, Karume K, Ngera M, Bisimwa M, Mushagalusa NG. 2012. Aspects of the physico-chemical characteristics of rivers in Kahuzi-Biega National Park, Democratic Republic of Congo. Journal of Environmental Protection, 3(11): DOI: 10.4236/jep. 2012.311175.

Bakundukize C, Van Camp M, Walraevens K. 2011. Estimation of groundwater recharge in Bugesera region (Burundi) using soil moisture budget approach. Geologica Belgica, 14(1-2): 85-102.

Ben Bouih H, Nassali H, Leblans M, Srhiri A. 2005. Contamination en métaux traces des sédiments du lac Fouarat (Maroc). Afrique Sciences, 1(1): 109-125.

Bliefert C, Perraud R. 2001. Chimie de l'environnement: Eau, Air, Sols, Déchets. Deboeck Université: Paris; 477.

Brion N, Nzeyimana E, Goeyens L, Nahimana D, Baeyens W. 2006. Inorganic nitrogen Uptake and river inputs in Northern Lake Tanganyika. Journal of Great Lakes Research, 32(3) 553-564. doi: http://dx.doi.org/10.3394/0380133032[553:INUARI]2.0.CO;2.

Cox TS, Bender M, Picone C, Van Tassel DL, Holland JB, Brummer EC, Zoeller BE, Paterson AH, Jackson W. 2002. Breeding Perennial Grain Crops. Critical Reviews in Plant Sciences, 21(2): 59-91. 
Downing LA, SB Watson, E Mc Cauley. 2001. Predicting cyanobacteria dominance in lakes. Canadian Journal of Fisheries and Aquatic Sciences, 58: 1905-1908.

Drieu O, Hakizimana G, Irimbere A, Musanisoni C, Tshibangu K, Kamalebo M, Mbemba M, Bwebwa D, Chitamwebwa DBR, Mwape LM. 2000. Lutte contre la pollution et autres mesures visant à protéger la iodiversité du Lac Tanganyika (RAF/92/G32).

Dudgeon D, Angela HA, Gessner MO, Kawabata ZI, Knowler DJ, Lévêque C, Naiman RJ, Prieur RAH, Soto D, Stiassny MLJ, Sullivan CA. 2006. Freshwater biodiversity: importance, threats, status and conservation challenges. Biol. Rev., 81:163-182. DOI: 10.1017/S1464793105006950.

Dufayt O, Nameche Th, Vasel JL, Edeline F. 1994. Une méthode simple de mesure de débit dans les chenaux à écoulement libre, système à injection des bulles. Fondation Universitaire Luxembourgeoise, Université de Liège.

Houssou AM, Agadjihouédé H, Montchowui E, Bonou CA, Lalèyè P. 2015. Structure and seasonal dynamics of phytoplankton and zooplankton in Lake Azili, small Lake of the pond of River Ouémé, Benin. International Journal of Aquatic Biology, 3(3): 161-171.

Jen O. 2002. The perceived Environmental Impact of Car Washing. RamseyWashington Metro Watershed District, 9.

Khalaf G, Slim K, Saad Z, Nakhlé K. 2007. Evaluation de la qualité biologique des eaux du Nahr el Jaouz (Liban) : application des méthodes indicielles. Bull. Mens. Soc. Linn. Lyon., 76(9-10) : 255- 268.

Kotak BG, Lam AKY, Prepas EE, Hurdley SE. 2000. Role of chemical and physical variables in regulating microcystin-LR concentration in phytoplankton of eutrophic lakes. Canadian Journal of Fisheries and Aquatic Sciences, 57: 1584-1593.
Leclercq L. 2001. Les eaux courantes : caractéristiques et moyens d'étude, dans Les zones humides. Actes des colloques organisés en 1996 par le Ministère de la Région Wallonne dans le cadre de l'Année Mondiale des Zones Humides, Jambes, Région Wallonne, DGRNE. 6782.

Li M, Xie GQ, Dai CR, Yu LX, Li FR, Yang SP. 2009. A study of the relationship between the water body chlorophyll a and water quality factors of the off coast of Dianchi Lake. Yunnan Geographic Environment Research, 21(2): 102-106.

Majaliwa JGM, Magunda MK, Tenywa MM. 2004. Non point pollution loading in a selected micro catchment of the Lake Victoria basin. In: Proceedings of the Ninth International Symposium on river sedimentation. Yinchang. China, 2206$2211 \mathrm{p}$.

Mohammad MJ, Mazareh N. 2003. Changes in soil fertility parameters in response to irrigation of forage crops with secondary treated wastewater. Communication in Soil Science and Plant Analysis, 34(910): 1281-1294.

Nassali H, Ben Bouih A, Srhiri MD, Hahbi. 2005. Influence des rejets des eaux usées sur la composition des eaux de surface et des sédiments superficiels du Lac Merja Fouarate au Maroc. Afrique Science, 1(1): 145-165.

Niyungeko C. 2011. Evaluation de l'efficacité épuratoire de la station de lagunage de Buterere. Mémoire présenté en vue de l'obtention d'un Diplôme de Master Complémentaire en Sciences de l'Environnement, Facultés des Sciences, Université du Burundi, 71p.

Nsavyimana G. 2014. Modélisation des processus physiques et biologiques dans des fosses septiques et voies de valorisation des boues de vidange: Application à Bujumbura-Burundi. Thèse de doctorat. Université de Liège.

Ntakiyiruta P, Nahimana D, Ntakimazi G. 2017. Contribution à l'estimation quantitative des nutriments apportés par 
la rivière Ntahangwa au lac Tanganyika. Afrique Science, 13(1): 360 - 370.

Pitt R, Williamson D, Voorhees. 2005. Review of historical street dust and dirt accumulation and wash off data. Dans Effective modeling of urban systems, Monograph 13, James, Irvine, McBean et Pitt (eds), CHI, Guelph, Ontario.

Rodier J. 2005. Analyse de l'Eau: Eaux Naturelles, Eaux Résiduaires, Eaux de Mer: Chimie, Physicochimie, Biologie, Interprétation des Résultats. Dunod: Paris

Rodier J, Legube B, Merlet N. 2009. Analyse de l'Eau (9 $9^{\text {ème }}$ édn). Dunod: Paris.

Saad Z, Slim K, Khalaf G, Elsamad O. 2004. Impact des rejets des eaux résiduaires sur la qualité physico-chimique et algologique du Nahr Antélias. Bulletin de la Société Neuchâteloise des Sciences Naturelles, 127: 69-82.

Sala F, Osvaldo E, Stuart Chapin III, Juan J, Armesto, Berlow E, Bloombeld J, Dirzo R, Huber-Sanwald E, Laura F, Huenneke, Robert B, Jackson, AKinzig, Rik L, David M, Lodge, Harold A, Mooney, Martõ ON, LeRoy P, Martin T, Sykes, Brian H, Walker, Marilyn W, Diana H, Wall. 2000. Global Biodiversity Scenarios for the Year 2100. Science 287: 1770. DOI: 10.1126/Science. 287. 5459.1770.

Salgot M, Huertas E. 2006. Integrated Concepts for Reuse of Upgraded Wastewater, Guideline for quality standards for water reuse in Europe, AQUAREC, EVK1-CT-2002-00130, Work package 2, Deliverable D15, 128p. Sondergaard M, Jensen LP, Jeppensen E. 2003. Role of sediment and internal loading of phosphorus in shallow lakes. Hydrobiologia, 506-509 : 135-145.

Vindimian E. 2006. Problématique des substances à risque. In: Conférence Eau et Santé. Eaux pluviales et assainissement : nouvelles préoccupations sanitaires, GRAIE, Grand-Lyon et ASTEE. 10.10, Lyonilleurbanne, 55-73.

Wong THF. 2006. Australia Runoff Quality-A Guide to Water Sensitive Urban Design. Engineers Australia: Melboume.

Zébazé Togouet Sh. 2008. Eutrophisation et structure de la communauté zooplanctonique du Lac Municipal de Yaoundé. Thèse de Doctorat $\mathrm{PhD}$, Faculté des Sciences, Université de Yaoundé I Cameroun ,200p+annexe.

Zirirane D, Bagalwa JJ, Isumbisho $\mathrm{M}$, Mulengezi M, Mukumba I, Bora M, Mucheso JM, Lukamba A, Iragi G, Iregne B, Kibangu F, Kamangala R. 2014. Evaluation comparée de la pollution des rivières Kahuwa et Mpungwe par l'utilisation des macroinvertébrés benthiques. Vertigo. La Revue Electronique en Sciences de l'Environnement, 14(3). 